\title{
La construcción del conocimiento en la red: límites y posibilidades.
}

\author{
Begoña Gros Salvat \\ bgrosaub. edu \\ Universidad de Barcelona
}

\section{La dimensión social del conocimiento}

Las teorías tradicionales sobre el aprendizaje han centrado su estudio en la interacción que acontece entre la persona y su entorno. Las teorías han proporcionado a lo largo del tiempo respuestas más o menos parciales al fenómeno del aprendizaje y han ido proporcionando explicaciones relevantes que han supuesto una mejora del conocimiento de los factores que nos conducen a diseñar entornos que favorezcan el aprendizaje.

La introducción de las tecnologías de la información y la comunicación (TIC) estuvo, en sus inicios, centrada en la mejora de los procesos individuales de aprendizaje. En cierto modo, buena parte de los discursos pedagógicos que han acompañado a la introducción de la tecnología informática en la educación se han centrado en enfatizar los beneficios de las máquinas en tanto que permiten una mayor adaptación al ritmo individual de los estudiantes.

En la última década y, al mismo tiempo que se ha ido desarrollando de forma considerable las posibilidades de uso de las TIC para los aspectos comunicativos, los discursos sobre el aprendizaje en grupo, en colaboración se han ido incrementando. De este modo, las TIC ya no se contemplan como una herramienta de interés para el aprendizaje individualizado sino también como un soporte para el aprendizaje grupal y la creación conjunta de conocimiento.

La tecnología está proporcionando herramientas de mucho interés que permiten crear espacios de comunicación, sistemas de documentos compartidos, de escritura grupal, de discusión a través de foros virtuales, etc. Sin embargo, la tecnología no crea la comunicación ni el aprendizaje. El aprendizaje a través de entornos colaborativos supone un reto importante ya que introduce formas de trabajo muy diferentes a las que se están utilizando en la mayoría de las instituciones de todos los niveles educativos. No se trata sólo de que los estudiantes aprendan a partir de un modelo colaborativo sino que también las instituciones aprendan ya que la dimensión social del conocimiento no alcanza sólo a la persona sino también a la propia organización.

Las teorías sobre el aprendizaje han comenzado a considerar la importancia de las interacciones sociales entre las personas actuando en un mundo social. La teoría de la cognición situada representa una de las tendencias actuales más representativa y heredera de las teorías de la actividad sociocultural. Toma como punto de referencia los trabajos de Vygotsky (1978) y de autores como Leontiev (1978) y Luria (1987) y más recientemente, los trabajos de Rogoff (1993), Lave (1997), Bereiter (1997), Engeström y Cole (1997), Wenger (2001), por citar sólo algunos de los más conocidos en el ámbito educativo. Se considera que el aprendizaje es una actividad situada en un contexto que la dota de inteligibilidad, según la cual la descontextualización del aprendizaje es imposible, puesto que toda adquisición de conocimiento está contextualizada en algún tipo de actividad social.

La aproximación antropológica, reflejada en el trabajo de Jean Lave y Etienne Wenger, se centra en la situacionalidad del significado en las comunidades y en lo que representa aprender en función de formar parte de una comunidad. Este cambio en la unidad de análisis, desde el contexto de los 
individuos al contexto de la comunidad, conduce a un cambio en el que se entiende el aprendizaje como "el desarrollo de una identidad como miembro de una comunidad y llegar a tener habilidades de conocimiento como parte del mismo proceso" (Lave y Wenger, 1991).

El aprendizaje supone la partipación en una comunidad y deja de ser considerado como la adquisición de conocimientos por individuos para ser reconocido como un proceso de participación social. Este proceso se denomina de participación periférica legítima, puesto que el nuevo participante, que se mueve de la periferia de la comunidad hacia el centro, llegará a ser más activo y a estar más comprometido con la cultura y, por ello, asumirá una nueva identidad.

Para estos autores, el aprendizaje es el resultado de formar parte de comunidades. Desarrollan el concepto comunidad de práctica para manifestar la importancia de la actividad como nexo entre el individuo y la comunidad, así como de las comunidades para legitimar las prácticas individuales.

Desde la perspectiva de esta teoría del aprendizaje social, el significado y las identidades son construidos en las interacciones, mientras que la construcción de estos significados e identidades es influenciada por el contexto en el que se inscriben. En las comunidades de práctica no hay separación entre el desarrollo de la identidad y el desarrollo del conocimiento, ambos interactúan recíprocamente mediante el proceso de participación periférica legítima en el contexto de la comunidad.

Bajo esta perspectiva, todos los autores parten de una fuerte crítica a la manera cómo la institución escolar intenta promover el aprendizaje. En particular, cuestionan la forma en que se enseñan aprendizajes abstractos y descontextualizados, conocimientos poco útiles y de relevancia social limitada (Díaz Barriga y Hernández, 2002). Es decir, en las escuelas se privilegian las prácticas educativas artificiales, en las cuales se manifiesta una ruptura entre el saber qué y el saber cómo y donde el conocimiento se trata como si fuera neutral, ajeno, autosuficiente e independiente de las situaciones de la vida real o de las prácticas sociales de la cultura a la que se pertenece.

Una situación educativa, para efectos de su análisis e intervención, requiere concebirse como un sistema de actividad cuyos componentes incluyen (Engeström 1987):

- El sujeto que aprende.

- Los instrumentos utilizados en la actividad, especialmente los de tipo semiótico.

- El objeto a apropiarse u objetivo que regula la actividad (saberes y contenidos).

- Una comunidad de referencia en que la actividad y el sujeto se insertan.

- Normas o reglas de comportamiento que regulan las relaciones sociales de esa comunidad.

- Reglas que establecen la división de tareas en la misma actividad.

Bajo este enfoque, la construcción del conocimiento no es una empresa nada fácil ya que requiere de unas condiciones de trabajo muy bien determinadas, unas herramientas que favorezcan los procesos y un seguimiento continuo.

\section{El aprendizaje colaborativo mediado por ordenador.}

El aprendizaje colaborativo mediado por ordenador supone una posible vía de utilización que ofrece varias ventajas y que expresa dos ideas importantes. En primer lugar, la idea de aprender de forma colaborativa, con otros, en grupo. No se contempla al aprendiz como persona aislada sino en interacción con los demás. Se parte de la idea de la colaboración y, por consiguiente, compartir objetivos y distribuir responsabilidades son formas deseables de aprendizaje. Además, se enfatiza el papel del ordenador como elemento mediador que apoya este proceso. En este sentido, el software utilizado tiene que favorecer a los procesos de interacción y de solución conjunta de los problemas.

Crook señala que "los estudios sobre el aprendizaje cooperativo contribuyen a definir una estructura 
de motivación y de organización para un programa global de trabajo en grupo, mientras los estudios sobre el aprendizaje colaborativo se centran en las ventajas cognitivas derivadas de los intercambios más íntimos que tienen lugar al trabajar juntos" (1998: 168).

Driscoll y Vergara (1997: 91), explicitan que para que exista un verdadero aprendizaje colaborativo no sólo se requiere trabajar juntos sino que hay que cooperar para alcanzar una determinada meta que no podría lograrse de forma individual. En este sentido, indican la existencia de cinco elementos básicos que caracterizan el aprendizaje colaborativo:

1. Responsabilidad individual: todos los miembros del grupo son responsables de su desempeño individual dentro del grupo.

2. Interdependencia positiva: los miembros deben depender los unos de los otros para lograr una meta común.

3. Habilidades de colaboración: las habilidades necesarias par que el grupo funcione de forma efectiva, como el trabajo en equipo, la solución de conflictos, el liderazgo, etc.

4. Interacción promotora: los miembros del grupo interactúan para desarrollar relaciones interpersonales y establecer estrategias efectivas de aprendizaje.

5. Proceso de grupo: el grupo reflexiona en forma periódica y evalúa su funcionamiento, efectuando los cambios necesarios para incrementar su efectividad.

En esta misma línea, Puntambekar y Young (2003) establecen que las dimensiones fundamentales del aprendizaje colaborativo mediado por ordenador son las siguientes:

- La naturaleza de las metas de los aprendices (conocimientos previos, etc)

- Articulación de los objetivos compartidos. La evidencia de las metas compartidas debería ser el primer factor y la base del aprendizaje colaborativo mediado por ordenador. De las metas compartidas hay que ir al entendimiento compartido.

- Papel del profesor

- Naturaleza del contexto. Usar una misma herramienta en diferentes contextos muestra que hay efectos diferentes

Estas condiciones, fáciles de describir, son precisamente el elemento más complejo para el desarrollar en la práctica.

\section{Características de los mediadores para favorecer el aprendizaje.}

El cambio que supone pasar de un modelo centrado en la transmisión de información a un diseño centrado en el futuro usuario es importante. En esta línea son numerosas las propuestas que han surgido en los últimos años: el aprendizaje basado en la resolución de problemas, el aprendizaje colaborativo, el aprendizaje centrado en escenarios, los entornos constructivistas de aprendizaje, la creación de micromundos, el aprendizaje situado, las comunidades de aprendizaje, etc. Todas estas propuestas buscan un uso de la tecnología como favorecedora y mediadora del aprendizaje y todas se caracterizan por los siguientes aspectos:

- Un diseño centrado en el estudiante, enfatizando las actividades a realizar sobre los contenidos a transmitir.

- Un diseño en el que se concede mucha importancia al contexto de aprendizaje por lo que se trata de proponer tareas lo más realistas posibles. 
- La tecnología interpretada como una herramienta mediadora. El diseño de entornos virtuales para el aprendizaje debe ser analizado dentro del contexto de cambio y evolución de la sociedad.

Denominamos entorno virtual de aprendizaje a la creación de materiales informáticos de enseñanzaaprendizaje basados en un sistema de comunicación mediada por el ordenador. En el momento de diseñar un entorno virtual de aprendizaje es importante establecer una clara diferenciación entre el diseño de un portal o web informativa y un entorno virtual. En este sentido, la proliferación de documentos y publicaciones sobre este tema está llevando a una cierta confusión entre ambos términos y, en ocasiones, se aplican diseños de usabilidad que no siempre han estado contrastados como estrategias didácticas correctas. Es preciso diferenciar claramente entre una organización de la información para favorecer el acceso a la misma de un sistema formativo.

Consideramos que el diseño de un entorno para la formación debería tener en cuenta una serie de características específicas que proporcionen el medio a partir de las cuales nos debemos plantear su explotación o no según el caso concreto con que nos encontremos. Al igual que sucede con cualquier otro tipo de diseño formativo, lo más importante de un entorno de aprendizaje virtual no es la posibilidad técnica del medio sino las estrategias pedagógicas que hemos de utilizar. Por ello, consideramos que debemos tener presente lo especifico, el "valor añadido" proporcionado por el entorno.

En base a las aportaciones de Dillenbourg, (2000), podemos considerar que existen siete elementos básicos a tener en cuenta cuando hablamos del diseño de entornos virtuales para la formación

\subsection{Un entorno virtual de aprendizaje es un espacio diseñado con finalidades formativas.}

Como ya hemos mencionado previamente, el hecho de construir un sitio web más o menos estructurado no garantiza ningún tipo de aprendizaje. Por ello, el uso del concepto de entorno virtual de aprendizaje debe diferenciarse de un espacio informativo bien estructurado.

El diseño debe nutrirse principalmente de las investigaciones y aportaciones del diseño formativo y es necesario que la investigación avance mucho más para obtener un mejor conocimiento de la relación funcional entre cómo está estructurada y representada la información y cómo puede ser utilizada en actividades de aprendizaje e interacción. La gestión y organización del conocimiento, el uso de representaciones hipertextuales, la adquisición de información a través de simulaciones, etc. Todos estos medios son accesibles en un entorno virtual pero es preciso saber en qué momento utilizarlo en función de qué objetivos y qué tipo de aprendizajes queremos alcanzar.

\subsection{Un entorno virtual de aprendizaje es un espacio social.}

Un libro no puede ser descrito como un entorno de aprendizaje. Sin embargo, leer un libro en un seminario, discutirlo con los demás estudiantes, escribir un resumen, constituye un entorno de aprendizaje. De forma similar, un conjunto de páginas web no constituyen un entorno virtual de aprendizaje a menos que haya una interacción social sobre la información. Esto incluye comunicación sincrónica, asincrónica, la posibilidad de compartir espacios, etc. La noción de "espacio social" (Brown-.Duguid 2001) abre posibilidades muy interesantes que todavía deben ser exploradas ya que en la mayor parte de los usos actuales todavía queda muy restringido un uso enciclopédico de la información.

\subsection{El espacio social está representado explícitamente.}

La representación de la información en un entorno de aprendizaje virtual puede ser muy variada. Desde un espacio basado fundamentalmente en texto hasta la incorporación de simuladores en tres dimensiones. No obstante, el sistema de organización de la información es cada vez más hipertextual lo que otorga un papel más activo al usuario. Este sistema de organización y presentación facilita el papel activo del lector en el proceso de exposición a los contenidos, escogiendo los trayectos de su 
exploración que no habrán de ajustarse necesariamente a una secuencia lineal, y decidiendo el ritmo del proceso, así como el atractivo que supone el acceso al contenido presentado de forma estática (texto e ilustraciones) y dinámica (mediante sonido, animaciones, vídeo).

La percepción de telepresencia, de estar en un espacio virtual de aprendizaje, se debe a las sensaciones a las que da lugar la participación en el entorno hipermedia y a la posibilidad de relacionarse con otras personas que también acceden a él. El grado en el que se experimenta esta sensación depende del nivel de realismo que se consiga en la presentación de contenidos a través del propio entorno.

El proceso de decisión de movimientos del usuario en su exploración por la red - siguiendo las relaciones que conectan los distintos nodos en los que se estructura el hipertexto para buscar, seleccionar y recuperar información - proporciona una gran libertad de elección y un elevado control de los movimientos, superiores a los que confieren los medios convencionales y los sistemas telemáticos con interacción controlada y centralizada.

El aspecto clave no es la representación por si misma, sino qué hacen los estudiantes con la representación ya que el espacio social representado no es neutro. La "biblioteca", el "cibercafé", etc. condicionan el tipo de relación y comunicación de los estudiantes. Y, de hecho, se acostumbran a utilizar representaciones que tienen una correspondencia en el espacio habitual de clase como elemento de enlace con los nuevos entornos.

\subsection{Los estudiantes no sólo son activos sino también actores, co-construyen el espacio virtual.}

En un entorno virtual de aprendizaje, los estudiantes pueden ser también diseñadores y productores de contenidos. En este sentido, el papel es mucho más participativo y activo ya que puede contribuir con sus aportaciones, aumentar la base de conocimiento, reforzar enlaces, etc. En definitiva, el conocimiento es mucho más dinámico y cambiante.

\subsection{Los entornos virtuales de aprendizaje no están restringidos a la enseñanza a distancia, también pueden enriquecer la enseñanza presencial.}

A menudo se centra el estudio del diseño de los entornos virtuales en los campus virtuales y se sitúa en la enseñanza a distancia. Sin duda, la enseñanza a distancia se beneficia de forma importante de los nuevos medios de transmisión de información y comunicación pero también la enseñanza presencial. Por este motivo, el concepto de semi-presencialidad o bimodalidad se va extendiendo rápidamente y la enseñanza formal y las universidades están incorporando actividades formativas en la red como elemento complementario.

\subsection{Los entornos virtuales de aprendizaje integran diferentes tecnologías y también enfoques pedagógicos múltiples.}

No se pueden hacer comparaciones sobre las ventajas e inconvenientes de la enseñanza presencial o a distancia sin concretar el tipo de tecnología y enfoque pedagógico utilizado. Un entorno de aprendizaje virtual depende siempre de la variedad de herramientas que se utilizan y del tipo de modelo educativo desarrollado. En definitiva, un entorno virtual de aprendizaje integra una gran variedad de herramientas que apoyan las múltiples funciones: información, comunicación, colaboración, aprendizaje, gestión, etc.

\subsection{La mayoría de los entornos virtuales no excluyen los entornos físicos.}

El uso de un entorno virtual no excluye la utilización de otros tipos de materiales. A menudo aparecen controversias sobre las ventajas e inconvenientes de los medios tradicionales respecto a las tecnologías actuales. Sin embargo, el uso de unos medios no anula a los otros y, generalmente unos se apoyan en los otros. Por este motivo, podemos diseñar un entorno virtual con material en red pero complementado con la lectura de libros, artículos, utilización de películas, etc. 
Como ya hemos señalado, la capacidad de Internet y de las tecnologías multimedia expone al estudiante a numerosas fuentes de información entre los cuales debe realizar elecciones. El cambio fundamental de orientación educativa modifica la función del profesor como única fuente experta de información para muchos estudiantes, a una situación formativa centrada en el alumno en donde cada aprendiz está en contacto con muchas fuentes de información. Especialmente en la red, la riqueza de la información tiene numerosas ventajas, pero también somete al aprendiz a muchas fuentes inútiles y falsas de información que cada estudiante debe evaluar, seleccionar y adaptar. Por ello, el papel del profesor cambia y pasa de ser un proveedor de información a un facilitador que ayuda a seleccionar la información, propone tareas, problemas o proyectos de interés, dirige al alumno para lograr productos significativos y construir el conocimiento.

\section{Investigaciones sobre la construcción del conocimiento en red.}

El estado de las investigaciones en este campo es muy dinámico y existen en la actualidad mucho interés en el mismo. Sin embargo, es todavía bastante prematuro establecer conclusiones claras sobre algunas de las líneas iniciadas. No obstante, intentaremos establecer algunas de las cuestiones más habituales que abren líneas de trabajo e investigación y que, además, son introducidas por los diversos artículos que componen este monográfico.

Las investigaciones se pueden centrar en cuatro áreas: el estudio de la interacción, la intervención del profesor en el espacio colaborativo, la construcción colaborativa del conocimiento y las herramientas mediadoras.

\subsection{Estudios sobre la interacción.}

La interacción que se produce en un entorno colaborativo virtual es diferente que la producida en un entorno presencial, cara a cara. Obviamente, se comparte un espacio común, hay un encuentro con los otros pero las características específicas de este espacio está condicionando las formas de relación e interacción.

Para que haya un aprendizaje no se trata sólo de intercambiar mensajes, de conversar. Hay que lograr un diálogo que favorezca la elaboración del conocimiento. Ahora bien, qué papel tiene el diálogo en el proceso interactivo. Se aprende del diálogo, aprendemos a dialogar, qué tipo de argumentaciones se producen en el diálogo, cómo se promueve el aprendizaje a través del diálogo. Estas son algunas de las cuestiones más relevantes que aparecen en las investigaciones sobre el proceso interactivo durante la colaboración.

Crook (1996) considera que el estudio del papel del diálogo en el aprendizaje y la observación de los diálogos es fundamental para poder diseñar entornos de aprendizaje que lo favorezcan. Para ello, propone la creación de entornos experimentales que permitan enlazar las teorías sobre el aprendizaje con el análisis de las interacciones.

Baker (2003) considera que lo importante es analizar la interacción argumentativa que es un tipo de diálogo en el que se combina la argumentación y la negociación del significado. La argumentación esta formada por los tipos de relación entre la solución de lo que se está discutiendo y las fuentes de conocimiento. Es un diálogo en el que se combinan los pros y contras de las contribuciones y se requiere un nivel importante de fundamentación durante el proceso de discusión. La negociación del significado se produce durante el proceso de solución del problema, de manera que se genera un espacio entre el problema planteado y su solución.

En definitiva, para que realmente haya cooperación se requiere que los sujetos adquieran un marco común de referencia que les permita negociar y comunicar sus puntos de vista personales y realizar inferencias en una base común. La negociación del conocimiento puede darse sobre la solución del problema, las fuentes, los significados, etc. La cuestión estriba en la relación entre la negociación del conocimiento y aprendizaje mediante la colaboración. 
Un aspecto complejo del análisis del proceso interactivo durante la colaboración a través del ordenador surge a partir de la necesidad de establecer categorías que permitan trabajar el análisis de los mensajes. El reto actual se sitúa precisamente en el estudio del contenido y del proceso de la interacción. Este es uno de los temas que se plantea en el artículo de A. Lazaro quien establece las condiciones y marco más adecuado para la creación de la comunidades de aprendizaje en la red.

\subsection{Moderación de los espacios colaborativos.}

Salmon (2000) empleó por primera vez el término "e-moderating" para describir la extensa variedad de funciones y destrezas que el profesor o formador en línea necesita adquirir. Apoyar el aprendizaje virtual por medio de conferencias, tanto en tiempo real (sincrónicas) como en el tiempo diferido (asíncronas), requiere del moderador un amplio abanico de habilidades comparado con las tareas que se realizan en una situación de enseñanza presencial. De ahí que la función del profesor necesita ampliarse de modo que incluya la intervención en entornos virtuales para hacer frente al desarrollo y potencial de los nuevos entornos de aprendizaje.

Sea cual sea el nivel de sofisticación tecnológica, la persona que aprende en línea no desea prescindir del apoyo humano en su aprendizaje. Mucho se ha escrito ya sobre las nuevas tecnologías y su potencial, pero no tanto acerca de lo que los facilitadores humanos deben hacer. Si bien un número cada vez mayor de personas aprenden hoy día en un entorno virtual, son pocos los profesores que han aprendido ellos mismos por este medio. Consecuentemente, la moderación no es un conjunto de habilidades que la mayoría de los profesores hayan adquirido de manera vicaria a través de la observación de sus profesores al tiempo que ellos mismos realizaban su aprendizaje. Muchos profesores tienden, de forma compresible, a suponer que la función del moderador tiene simplemente que ver con el dominio de un nuevo programa de software o de destrezas en el uso del ordenador. Pero la situación real es muy diferente y hay que crear estrategias que permitan los diversos grados de cooperación. Tres artículos abordan el tema: P. Colas y J. De Pablos proponen una técnica para la formación del profesorado basada en las redes de aprendizaje virtual. Juan Silva analiza el papel del tutor en un curso de formación continua para docentes. M. Adrian y B. Gros describen una experiencia basada en el análisis de algunos cursos semi-presenciales que utilizan foros electrónicos para el trabajo colaborativo.

\subsection{Mediación.}

El software diseñado para el trabajo colaborativo y la comunicación en línea va aumentando de forma considerable pasando de los diseños centrados en la interacción personal a los centrados en la interacción grupal. La selección de la herramienta más adecuada en función de los objetivos que deseamos alcanzar hace necesario un estudio más exhaustivo del valor de las diferentes herramientas y características más apropiadas para cada caso. No es lo mismo la utilización de una comunicación asincrónica a través de un forum o de una comunicación inmediata a través de un chat.

Las diferencias en cuanto modos de comunicación y las herramientas apropiadas son tratados por I. Alvarez y B.Kilbourn.

\subsection{Construcción colaborativa del conocimiento.}

El enfoque de las investigaciones alrededor de este tema tiene por objeto el estudio de la forma en que puede lograr un modelo de enseñanza basado en la comprensión y contrición compartida del conocimiento. Scardamalia y Bereiter se han convertido en los autores canadienses más influyentes entre los investigadores del aprendizaje colaborativo mediado por ordenador. Estos autores, han desarrollado una teoría sobre la construcción colaborativa del conocimiento a la vez que han elaborado herramientas tecnológicas acordes con dicho modelo teórico y han impulsado numerosas investigaciones aplicadas a la escuela considerada ésta como una comunidad de aprendizaje.

La aportación en esta monografía de M. Scardamalia nos introduce en este número monográfico y nos 
lleva a la reflexión sobre cómo debe transformarse la educación en la sociedad del conocimiento.

\section{Referencias}

Baker, M. (2003). Computer supported collaborative learning in the space of

debate. Designing for change in networked learning environments. B.-L. Wason,

Hoppe,U. Dordrecht, Kluwer Academic Publishers: 11-20.

Barad,S-Duffy,T (2000). From Practice Fields to Communities of Practice. Jonassen, D-Land,S (Eds) (2000). Theoretical Foundations of Learning Environments. Mahwah: Lawrence Erlbaum Associates. 25-56.

Bereiter, C. (1997). Situated cognition and how to overcome it. En D. Kirshner y J. A. Whitson (Eds.), Situated cognition. Social, semiotic and psychological perspectives (pp. 281-300). Mahwah, NJ: Lawrence Erlbaum.

Bereiter, C., \& Scardamalia, M. (2000). Process and product in Problem-Based Learning (PBL) research. In D. H. Evensen, and C.E. Hmelo (Eds.), Problem-Based Learning, a research perspective on learning interactions (pp.185-195). Mahwah, NJ: Lawrence Erlbaum Associates.

Brown, J.S., Collins, A. \& Duguid, P. (1989). Situated cognition and the culture of learning. Educational Researcher, 18(1), 32-42.

Brown,J.S-Duguid,P (2001). La vida social de la información. Buenos Aires: Prentice Hall.

Cabero,J (2001). Tecnología educativa. Diseño y utilización de medios en la enseñanza. Barcelona: Paidós.

Crook, C. (1996). Computers and the collaborative experience of learning. New York: Routledge.

Daniels, H. (2003). Vygotsky y la pedagogía. Barcelona: Paidós.

Debray, R (2001). Introducción a la mediología. Barcelona: Paidós.

Díaz Barriga, F. (2003). Cognición situada y estrategias para el aprendizaje significativo. Revista Electrónica de Investigación Educativa, 5 (2). [http://redie.ens.uabc.mx/vol5no2/contenidodiazbarriga.html]

Díaz Barriga, F. y Hernández, G. (2002). Estrategias docentes para un aprendizaje significativo. Una interpretación constructivista (2 ${ }^{\mathrm{a}}$. ed.). México: McGraw Hill.

Dillenbourg, P (2000). "virtual learning environments". Workshop on virtual learning. [http://tecfa.unige.ch/tecfa/publicat/dil-papers-2]

Engeström,E (1987). Learning by expanding: an activity-theoretical approach to developmental research. Helsinki: Orienta-Konsultit

Engeström, Y. y Cole, M. (1997). Situated cognition in search of an agenda. En D. Kirshner y J. A. Whitson (Eds.). Situated cognition. Social, semiotic and psychological perspectives (pp. 301-309). Mahwah, NJ: Lawrence Erlbaum.

Gros, B (2002) "Constructivismo y diseño de entornos virtuales de aprendizaje". Revista de Educación. 328, 225-247 
Harasin,L et al (2000). Redes de aprendizaje. Guía para la enseñanza y el aprendizaje en red. Barcelona: Gedisa.

Jonassen, D (1999). Designing Constructivist Learning Environments. Reigeluth, Ch (Ed). Instructional-Design Theories and Models. A New Paradigm of Instructional Theory. New Jersey: Lawrence Erlbaum Associates, 215-240.

Jonassen, D-Land,S (Eds) (2000). Theoretical Foundations of Learning Environments. Mahwah: Lawrence Erlbaum Associates.

Jonassen, D-Peck,K-Wilson,B (1999). Learning with Technology. New Jersey: Prentice Hall.

Lave, J. (1997). The culture of acquisition and the practice of understanding. En D. Kirshner y J. A. Whitson (Eds.), Situated cognition. Social, semiotic and psychological perspectives (pp. 17-35). Mahwah, NJ: Lawrence Erlbaum.

Lave, J. y Wenger, E. (1991). Situated learning: Legitimate peripheral participation. Cambridge: Cambridge University Press.

Lave,J-Wenger,E (1991). Situated Learning. Legitimate peripheral participation. Cambridge: Cambridge University Press.

Leontiev, A. (1978). Actividad, conciencia y personalidad. Buenos Aires: Ciencias del Hombre.

Luria. A. R. (1987). Desarrollo histórico de los procesos cognitivos. Madrid: Akal.

Morin,E (1999). La tête bien faite. Paris: Seuil.

Oliver, K (2000). Methods for Developing Constructivist Learning on the Web. Educational Technology, Nov-Dec, 5-18.

Oliver, R (2002). "Learning settings and activities". H. Adelsberger-B.Collis-J.M. Pawlowski (eds). Handbook on information technologies for education and training. Berlin: Springer Verlag, 199-212.

Palincsar, A. S. y Brown, A. L. (1984). Reciprocal teaching of comprehension-fostering and monitoring activities. Cognition and Instruction, 1, 117-175.

Paquette, G (2002). "Designing virtual learning centers". H. Adelsberger-B.Collis-J.M. Pawlowski (eds). Handbook on information technologies for education and training. Berlin: Springer Verlag, 249-272.

Posner, G. (1998). Enfoque de proyectos. En G. Posner, Análisis del currículo (pp. 181-190). Santafé de Bogotá: Mc Graw Hill.

Reigeluth, Ch (Ed) (1999). Instructional-Design Theories and Models. A New Paradigm of Instructional Theory. New Jersey: Lawrence Erlbaum Associates.

Rogoff, B. (1993). Aprendices del pensamiento. El desarrollo cognitivo en el contexto social. Barcelona: Paidós.

Salomon, G. (1993). No distribution without individuals' cognition: A dynamic interactional view. In G. Salomon (Ed.), Distributed cognitions. (pp 111-138). New York: Cambridge University Press.

Salmon, G (2000). E-moderating. London: Open University Press.

Scardamalia, M., \& Bereiter, C. (1994). Computer support for knowledge-building Communities. 
Journal of the Learning Sciences, 3(3), 265-283.

Scardamalia, M. y Bereiter, C. (1991). Higher levels of agency for children in knowledge building: a challenge for the design of new knowledge media. The Journal of the Learning Sciences, 1(1), 37-68.

Vygotsky, L. (1986). Pensamiento y lenguaje. Buenos Aires: La Pléyade.

Vygotsky, L. (1988). El desarrollo de los procesos psicológicos superiores. México: Grijalbo.

Wenger,E (2001). Comunidades de práctica. Aprendizaje, significado e identidad. Barcelona: Paidós.

Wilson, B.G., (1995). Metaphors for instruction: Why we talk about learning environments. Educational Tecnology, Special Section: Constructivist learning environments. Sept-Oct, 25-30.

Wilson,B-Madsen,K (2000). Situated Cognition in Theoretical and Practical Context. Jonassen, DLand,S (Eds) (2000). Theoretical Foundations of Learning Environments. Mahwah: Lawrence Erlbaum Associates. 57-88.

(C) Ediciones Universidad de Salamanca 